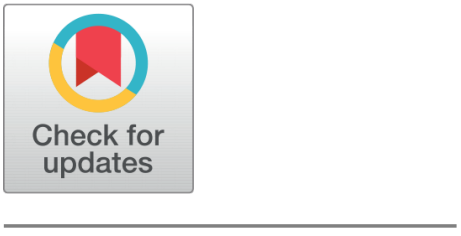

G OPEN ACCESS

Received: 03.12.2021

Accepted: 12.12 .2021

Published: 31.01 .2022

Citation: Bajpai V, Kumar V, Chouhan K, Basita A, Vasistha P, Kumar R (2022) Analysis of Settling and RCS Response of Copper Coated Carbon and its Comparison with Aluminium Fibres. Indian Journal of Science and Technology 15(2): 91-96. https://doi.org/ 10.17485/IJST/v15i2.2138

* Corresponding author.

prashantvasistha@dl.drdo.in

Funding: None

Competing Interests: None

Copyright: (c) 2022 Bajpai et al. This is an open access article distributed under the terms of the Creative Commons Attribution License, which permits unrestricted use, distribution, and reproduction in any medium, provided the original author and source are credited.

Published By Indian Society for Education and Environment (iSee)

ISSN

Print: 0974-6846

Electronic: 0974-5645

\section{Analysis of Settling and RCS Response of Copper Coated Carbon and its Comparison with Aluminium Fibres}

\author{
Vivek Bajpai ${ }^{1}$, Verandra Kumar ${ }^{2}$, Kavita Chouhan ${ }^{3}$, Alok Basita ${ }^{4}$, \\ Prashant Vasistha ${ }^{*}$, Ravindra Kumar ${ }^{6}$ \\ 1 Technical Officer, Defence Laboratory Jodhpur, India \\ 2 Scientist D, Defence Laboratory Jodhpur, India \\ 3 Scientist E, Defence Laboratory Jodhpur, India \\ 4 Scientist E, Defence Laboratory Jodhpur, India \\ 5 Scientist G, Defence Laboratory Jodhpur, India \\ 6 Scientist E, Defence Laboratory Jodhpur, India
}

\section{Abstract}

Background/Objectives: The present research focuses on comparative studies of two different type of chaff fibres for their effectiveness as electronics counter measure. Chaffs are electronic counter measures used in creating false radar signature to deceive tracking radar systems. Many types of chaff payloads are there in use, but the major one is aluminium fibres. These chaffs are designed to cover radar frequency range from 2-18 GHz. However, in future, tracking radars will operate in higher frequency range and will have improved algorithms to mitigate chaff noise of certain Radar Cross Section (RCS) threshold. Methods: The researcher used two different chaff fibres and their corresponding RCS are measured in anechoic chamber for their comparative study. Findings: It is observed that the RCS of the copper coated carbon (CuC) fiber is nearly equal to that of aluminium fiber but due their thinner diameter packing density of chaff fibres within a specified chaff cartridge volume has increased thus resulting in more number of dipole per chaff payload. Larger number of dipoles will help in improving dynamic RCS of chaff payload as it is directly proportional to the number of dipoles. Moreover, the terminal velocity of copper coated carbon fibres is lesser than that of aluminium fibres has improved the persistent time for CuCfiberwhich is a desirable parameter for the effective performance of chaff payload. Novelty: In order to increase dynamic RCS threshold one needs to increase chaff fiber density and chaff cloud suspension time. Both these can be achieved by the development of innovative thin CuCchaff fibres. In this paper, RCS of light weight and thin copper coated carbon chaff fibres were studied in comparison with traditional aluminium fibres and their advantages over aluminium fibres are reported.

Keywords: Backscattered RCS; electrically conducting fiber; anechoic chamber; chaff; Settling Time 


\section{Introduction}

In today's war scenario, chaff plays an important role as an electronic counter measure to deceive tracking radar systems ${ }^{(1,2)}$. Chaffs are conducting dipoles which act as passive reflectors to give spurious returns in the field of view of tracking radar sensors $^{(3)}$. Currently used chaffs are aluminium fibres, silver coated nylon monofilaments, chopped aluminium foils, etc. Today, various radars operate mainly in $\mathrm{GHz}$ frequency range thereby making the size of chaff dipoles, which is actually half of the operating wavelength, in centimetre range. But in the future, radars will operate in high frequency range, thereby forcing to use shorter dipole lengths to attain minimum Radar Cross Section (RCS) to deceive and jam the tracking radars. With decrease in dipole length, it will also require increase packing density of chaff dipoles to achieve the minimum required RCS. Therefore, to achieve this higher packing density, the thickness of the chaff dipole needs to be thinner ${ }^{(4,5)}$.

Carbon fibres have advantages over existing chaff materials as these have high strength and are very thin and light and available with diameter of $\sim 7$ micron. The only disadvantage of the carbon fibres is that their resistance is thousand times higher than the existing conducting material (primarily aluminium) used in fabrication of chaff dipoles. However, this limitation can be overcome by using coating of a conducting material (copper) over the carbon with very little compromise with the stiffness and thickness properties of the carbon fibres.

RCS measurement of chaff fibers with different cut lengths are reported in (2). However comparison of RCS of two different chaff fibres are not done. In this paper, static Radar Cross Section (RCS) of both copper coated carbon fibres and aluminium fibres have been studied and various advantages of copper coated carbon fibres over aluminium fibres have been reported.

\section{Experiment Setup and Measurements}

\subsection{Radar Cross Section (RCS) and its measurement}

The RCS of an object is defined in the far field and is independent of the distance between the radar and the object. Formally, the RCS of an object is defined as the ratio of power received per steradian (Sr) by the RADAR after reflection from the target to the power density which was transmitted at the target $(\mathrm{St})^{(6)}$.

$$
\begin{gathered}
\sigma=\lim _{R \rightarrow \infty} 4 \pi r^{2}\left|\frac{S r}{S t}\right| \\
\sigma=4 \pi \lim _{R \rightarrow \infty} R^{2} \frac{\left|E_{S}^{2}\right|}{\left|E_{i}^{2}\right|}
\end{gathered}
$$

Where $\mathrm{E}_{i}$ and $\mathrm{E}_{s}$ represent the incident and scattered field at a range R from the object and $\sigma$ represents the RCS. The RCS is a typical frequency domain quantity and is a function of Frequency, Object configuration, Transmitter and receiver polarization and angular orientation of the object with respect to the incident field.

In the present study, time domain techniques are used. In the Time-Domain (TD), ultra wideband pulse of $\sim 30$ picosecond time duration is used as test signals and measurements are carried out over the frequency range of 2-18 GHz. RCS measurement of a target involves two main steps-firstly calibration of the system using standard targets of known RCS and then using calibrated RF system for RCS measurement of the target. In calibration, standard targets like a metallic sphere is used to calibrate the reflected power with its known RCS value. Then RCS measurement of a target involves background measurement in which signals are transmitted and received in the absence of a target in an anechoic chamber and then measurement of reflected power in the presence of the target is performed. Received power during background measurement is subtracted from the received power obtained in the presence of target. This background power subtraction removes clutter contributions from leakage between transmit and receive antennae, reflections from chamber walls and absorbers. After background subtraction, measured power is compared with respect to the RCS values of a standard target to get the RCS values of target directly in dBsm. For calibration purpose, sphere of diameter $20 \mathrm{~cm}$ is used as shown in Figure 1 (a). Figure 1(b) shows time domain system for monostatic RCS measurement of chaff fibres.

For a comparative study of RCS behaviour of different chaff dipoles of given samples, dipole arrays of fixed arrangements (horizontal and vertical separation) of predefined dipole cut length $(50 \mathrm{~mm})$ are prepared as it will help in evaluating effects of various terms such as mutual coupling, screening effects, resonant frequency variations, etc. Figure 2 shows the schematic and Figure 3 shows the actual prepared dipole array used in the experiments. Cut length of the chaff dipoles used is $50 \mathrm{~mm}$ both in the case of copper coated carbon dipoles and aluminium fibres. Diameter of the fibres used is listed in Table 1. Measurement results (Normalized RCS) of copper coated carbon fibres and aluminium fibres are shown in Figure 4. 


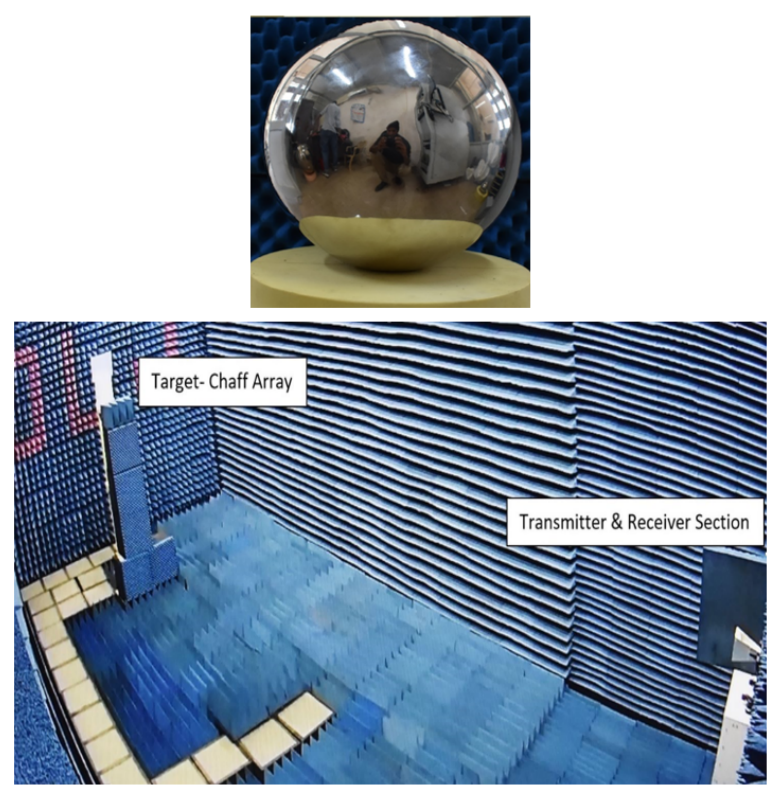

Fig 1. a) Standard Target Sphere (diameter $=20 \mathrm{~cm}$ ) for Calibration of the RF System b) RCS Measurement Set in Anechoic Chamber



Fig 2. Schematic Dipole Array arrangement

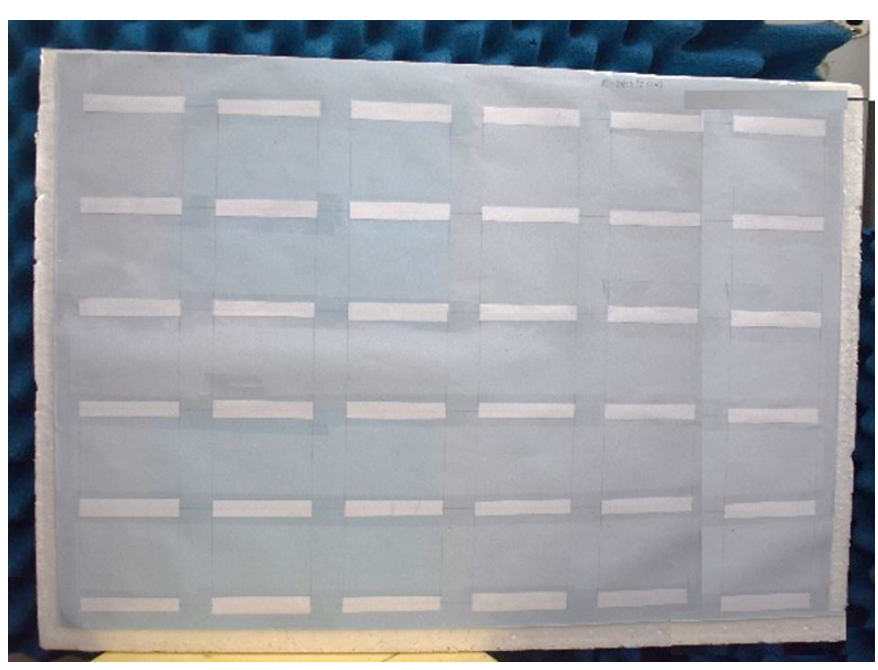

Fig 3. Actual Dipole Array arrangement 
Table 1. Diameters of the fibres used for measurements

\begin{tabular}{llll}
\hline S No. & Type of Fibre & Coating Thickness (um) & Diameter of Fibre (um) \\
\hline 1 & Aluminium & NA & 25 \\
2 & Aluminium & NA & 19.79 \\
3 & Aluminium & NA & 19.79 \\
4 & Aluminium & NA & 19.79 \\
5 & Aluminium & NA & 19.79 \\
6 & Copper Coated Carbon & 1.50 & 10.00 \\
7 & Copper Coated Carbon & 1.60 & 10.20 \\
8 & Copper Coated Carbon & 1.70 & 10.40 \\
9 & Copper Coated Carbon & 1.80 & 10.60 \\
10 & Copper Coated Carbon & 2.00 & 11.00 \\
\hline
\end{tabular}

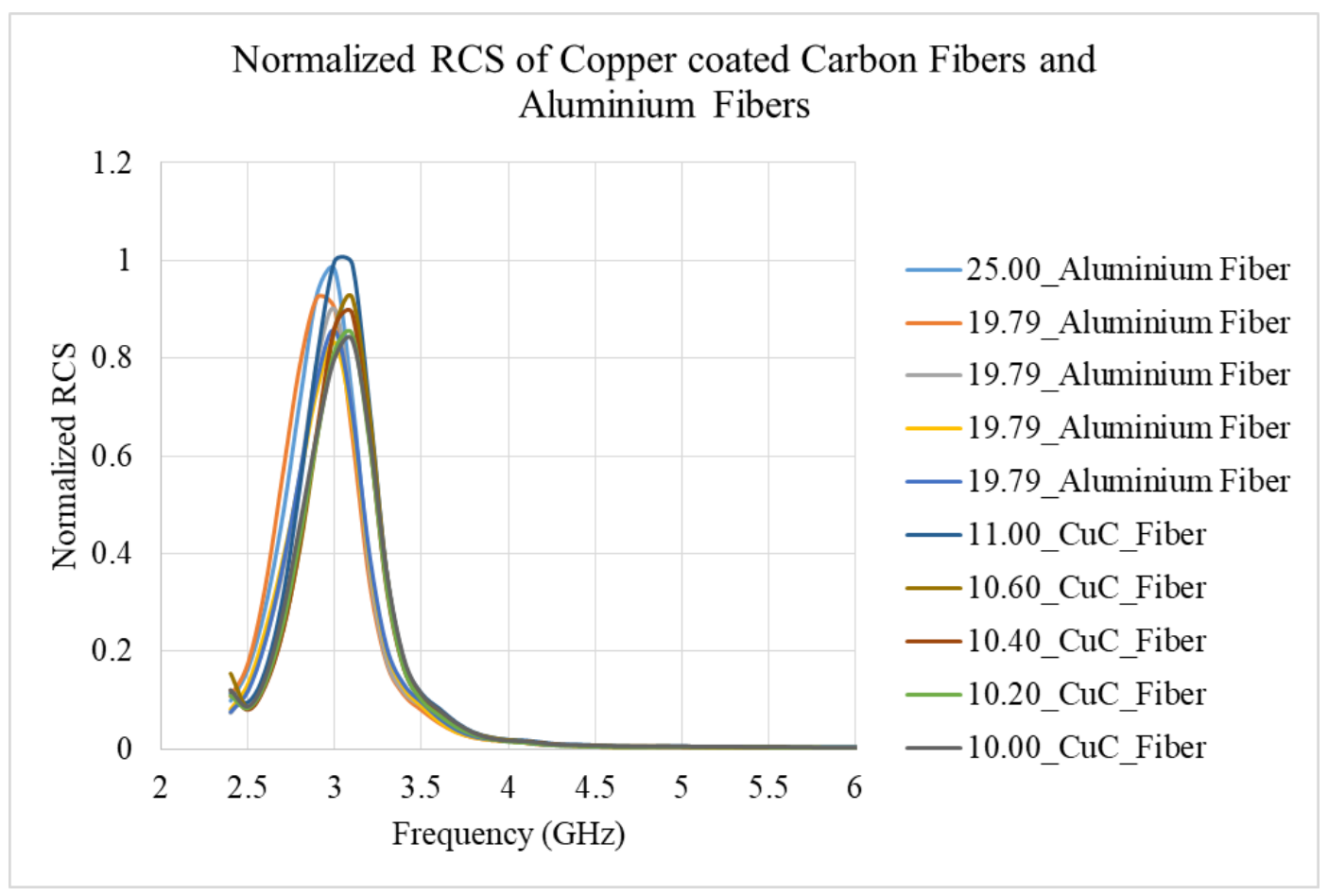

Fig 4. Comparison of Normalized RCS of Copper Coated Carbon $(\mathrm{CuC})$ and Aluminium fibres

\subsection{Effect of Terminal Velocity on persistence time of chaff cloud}

Generally, lower values of terminal velocity is desired for the chaff fibres. Lower terminal velocity results in higher settling time of the Chaff fiber thus fiber will remain suspended in air for longer duration.

Terminal velocity of a particle is defined as the steady speed reached by the particle when the particle is freely falling through a medium.

Mathematically, terminal velocity of a particle is given as ${ }^{(7)}$ -

$$
v_{t}=\frac{g * \rho * c^{2}}{6 * \mu}\left[\left(\ln \left(\frac{l}{c}\right)-\frac{3}{2}\right\} \sin ^{2}+\left(\ln \left(\frac{l}{c}\right)-\frac{1}{2}\right\}\right]
$$

$\mathrm{n}_{t}=$ terminal velocity

$\mathrm{m}=$ Viscosity of the fluid $=1.983 \times 10^{-5} \mathrm{Kg} / \mathrm{m} . \mathrm{s}$ for air

$\mathrm{g}=$ acceleration due to gravity

$\mathrm{Dr}=$ density difference between fiber and air 
$c=$ radius of fiber

l=length of fiber

As per equation (3), relationship between terminal velocity and chaff fiber equivalent weight and orientation is simulated in figure 5 .

\section{Terminal Velocity of $\mathrm{CuC}$ and Aluminium Fibers}

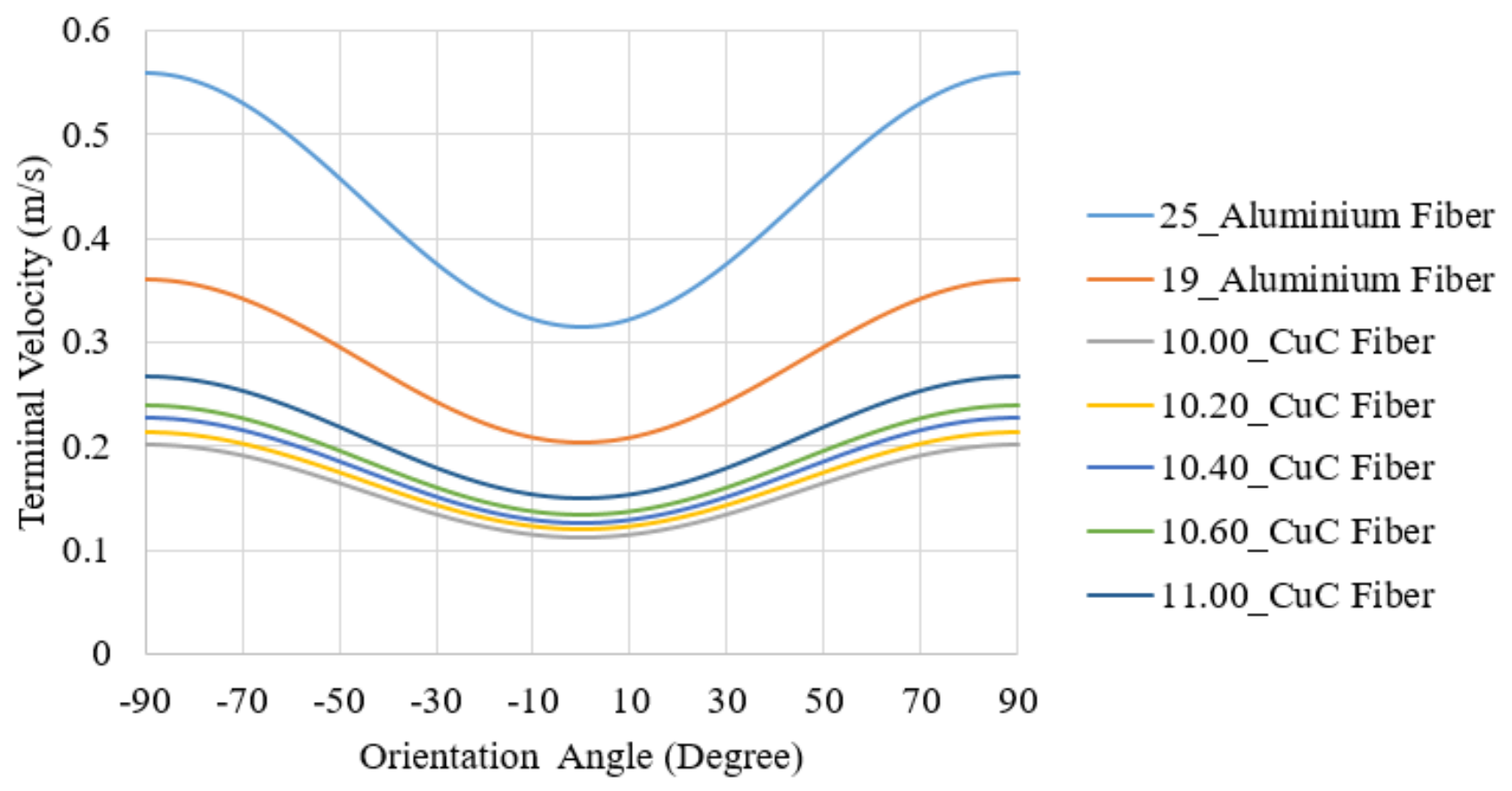

Fig 5. Terminal Velocity of Chaff fibres with different equivalent weight

\section{Results and Discussion}

It can be observed more the graph shown in figure 3 that the RCS of the copper coated carbon fiber which is thinner in diameter is nearly equal to that of aluminium fiber which is larger in diameter. RCS values so observed are in line with that reported in ${ }^{(2)}$. Backscattered RCS of chaff cloud is directly proportional to the number of the chaff dipole elements present in the chaff payload. Analytically ${ }^{(8)}$ the maximum RCS is given by

$$
\sigma_{(\max )}=\left(\mathrm{G}^{2} \lambda^{2}\right) / \pi
$$

Where $\mathrm{G}$ is the gain and $\lambda$ is the wavelength.

The maximum gain in far field for half wavelength antenna is given by

$$
\sigma_{\max }=0.856 \lambda^{2}
$$

For random orientation of dipole and averaging over $4 \pi$

$\sigma=0.172 \lambda^{2}$,

for $\mathrm{N}$ elements,

$$
\sigma=0.172 . \mathrm{N} \cdot \lambda^{2}
$$

From equation (6), RCS of dipoles $(\sigma)$ is directly proportional to the number of dipoles $(\mathrm{N})$.

Therefore, the number of dipole in a given volume of chaff cartridge will be more resulting in higher RCS for CuC fibres than that of aluminium fibres. 
It can be observed from Figure 5 that the terminal velocity of CuCfibers as per equation (3) is lesser as compared to aluminium fibers. Generally, terminal velocity of chaff fibers should be less for higher settling time. Although equivalent density of copper coated carbon fiber is slightly more compared to pure aluminium fibres but then also as per equation(3) the net effective terminal velocity of the CuCfiber is lesser as compared to pure aluminium fibres which will result in higher persistence time for chaff cloud.

\section{Conclusion}

The purpose of this study is to present a potential chaff material which is having RCS equal to that of tradition chaff material (Aluminium fibres). Copper Coated Carbon fibres are studied in comparison with that of traditional aluminium fibres. Two main advantages of $\mathrm{CuC}$ fibres are observed over traditional aluminium fibres. Firstly, $\mathrm{CuC}$ fibres are thinner than tradition aluminium fibres, therefore the number of $\mathrm{CuC}$ fibres will be more in predefined payload cartridge and compared to aluminium fibres, thus RCS of CuC fibres will be more as RCS is directly proportional to the number of fibres. Secondly, terminal velocity of $\mathrm{CuC}$ fibres at $0^{\circ}$ orientation is less than $0.149 \mathrm{~m} / \mathrm{s}$ for all CuC fibres and that of aluminium fibres is higher than $0.202 \mathrm{~m} / \mathrm{s}$ giving higher persistence time (a desirable quantity) for $\mathrm{CuC}$ chaff fibres. Thus, the study shows $\mathrm{CuC}$ have strong potential to be used as an alternate chaff material.

\section{References}

1) Zuo Y, Guo L, Liu W, Ding J. Jamming Efficiency Analysis Based on the Range Profile of Target with Chaff. IEEE Access. 2021;9:13573-13589. doi:10.1109/ACCESS.2021.3052076.

2) Kumar V, Vasistha P, Kumar R. RCS Simulation and Measurement Studies of Microwave Dipoles for Chaff Applications. International Journal of Advances in Microwave Technology. 2019;04(01):185-189. Available from: https://dx.doi.org/10.32452/ijamt.2019.185189. doi:10.32452/ijamt.2019.185189.

3) Mehmood S, Malik AN, Qureshi IM, Khan MZU, Zaman F. A Novel Deceptive Jamming Approach for Hiding Actual Target and Generating False Targets. Wireless Communications and Mobile Computing. 2021;2021:1-20. Available from: https://dx.doi.org/10.1155/2021/8844630. doi:10.1155/2021/8844630.

4) Bajpai V, Kumar V, Gadri KL, Singh AK, Mohammad N, Chouhan K, et al. Optimization of Coating Thickness of Conducting Material on Base Fiber to make it as a Radar Reflector. International Journal of Advances in Microwave Technology. 2021;06(01):247-253. Available from: https://dx.doi.org/10.32452/ ijamt.2021.247253. doi:10.32452/ijamt.2021.247253.

5) Gadri KL, Kumar V, Singh AK, Basita A, Vasistha P, Kumar R. Analysis of Effect of Surface Roughness of Electrically Conducting Fibre on its Backscattered RCS. International Journal of Advances in Microwave Technology. 2020;05(04):237-241. Available from: https://dx.doi.org/10.32452/ijamt.2020.237241. doi:10.32452/ijamt.2020.237241.

6) Knott EF. Radar Cross Section Fundamentals. In: EF K, editor. Radar Cross Section Measurements. Springer US. 1993;p. 1-26. Available from: http://link.sringer.com/book/1.1007/978-1-4684-9904-9.

7) Skolnik ML. Radar Cross Section of Targets. In: Skolnik ML. Introduction to RADAR Systems. 1981;p. 33-45. Available from: http://www.geo.uzh.ch/ microsite/rsl-documents/research/SARIab/GMTILiterature/PDF/Skolnik90.pdf.

8) Kumar V, Singh AK, Vasistha P, Kumar R. Dynamic RCS Prediction of Rapidly Blooming Chaff Cloud and its Validation using Measurement on Scaleddown. International Journal of Advances in Microwave Technology. 2018;3(4):170-175. Available from: https://dx.doi.org/10.32452/ijamt.2018.170175. doi:10.32452/ijamt.2018.170175. 